\title{
Unfolding codimension-two subsumed homoclinic connections in two-dimensional piecewise-linear maps.
}

\author{
D.J.W. Simpson \\ School of Fundamental Sciences \\ Massey University \\ Palmerston North \\ New Zealand
}

July 8, 2019

\begin{abstract}
For piecewise-linear maps, the phenomenon that a branch of a one-dimensional unstable manifold of a periodic solution is completely contained in its stable manifold is codimension-two. Unlike codimension-one homoclinic corners, such 'subsumed' homoclinic connections can be associated with stable periodic solutions. The purpose of this paper is to determine the dynamics near a generic subsumed homoclinic connection in two dimensions. Assuming the eigenvalues associated with the periodic solution satisfy $0<|\lambda|<1<\sigma<\frac{1}{\lambda}$, in a two-parameter unfolding there exists an infinite sequence of roughly triangular regions within which the map has a stable single-round periodic solution. The result applies to both discontinuous and continuous maps, although these cases admit different characterisations for the border-collision bifurcations that correspond to boundaries of the regions. The result is illustrated with a discontinuous map of Mira and the two-dimensional border-collision normal form.
\end{abstract}

\section{Introduction}

We begin by briefly reviewing related classical results for smooth maps. Let $\Gamma$ be a period- $n$ solution of a diffeomorphism on $\mathbb{R}^{2}$ with eigenvalues satisfying $0<|\lambda|<1<|\sigma|$. Then $\Gamma$ has one-dimensional stable and unstable manifolds; a tangential intersection between these is a homoclinic tangency. Generically the tangency is quadratic, to leading order. Homoclinic tangencies are global, codimension-one bifurcations that create chaos or represent a crisis where a chaotic attractor suddenly changes size 12 .

Now suppose $\xi \in \mathbb{R}$ is a parameter of the map and $\Gamma$ has a homoclinic tangency at $\xi=\xi^{*}$. Typically there exist single-round periodic solutions for values of $\xi$ near $\xi^{*}$. These have periods $k n+p$ for fixed $p$ and different values of $k$. If $\xi$ unfolds the tangency in a generic 
fashion and $|\lambda \sigma|<1$ at $\xi=\xi^{*}$, then there exists an infinite sequence of intervals $I_{k}$ such that the map has a stable period- $(k n+p)$ solution for all $\xi \in I_{k}$. As $k \rightarrow \infty$ the intervals converge to $\xi^{*}$ and are non-overlapping [4].

In two-dimensional parameter space homoclinic tangencies occur on curves. These curves may contain points where the tangency is codimension-two, such as the intersection between two homoclinic tangency curves associated with the same periodic solution. A determination of the dynamics near a codimension-two point is often helpful as it explains other curves of codimension-one bifurcations that may extend over large areas of parameter space [1, 6, 9].

This paper concerns piecewise-linear maps on a domain $\Omega \subset \mathbb{R}^{2}$ that we write as

$$
f(x, y)=\left\{\begin{array}{cc}
f_{1}(x, y), & (x, y) \in \Omega_{1}, \\
\vdots & \\
f_{m}(x, y), & (x, y) \in \Omega_{m} .
\end{array}\right.
$$

The regions $\Omega_{1}, \ldots, \Omega_{m}$ partition the domain $\Omega$, and each $f_{j}$ is assumed to be affine (linear plus a constant). We let $\Sigma$ denote the set of all $P \in \Omega$ for which every neighbourhood of $P$ intersects more than one $\Omega_{j}$. We assume $\Sigma$ is a finite union of smooth curves; it is the collection of switching manifolds of (1.1).

Now consider a period- $n$ solution of (1.1) that has no points in $\Sigma$ and eigenvalues satisfying $0<|\lambda|<1<|\sigma|$. Its stable and unstable manifolds are piecewise-linear, so cannot form a quadratic tangency. Instead they may form a homoclinic corner: locally one manifold is linear while the other has a kink. But unlike a homoclinic tangency, even if $|\lambda \sigma|<1$ close to a generic homoclinic corner there are no stable periodic solutions [16].

At a homoclinic corner, one orbit in the unstable manifold belongs to the stable manifold. It is a codimension-two phenomenon for two different orbits to have this property. But since (1.1) is piecewise-linear, this can imply all orbits in a branch of the unstable manifold belong to the stable manifold, Fig. 1. In this case the branch is completely contained within the stable manifold and we say it is subsumed.

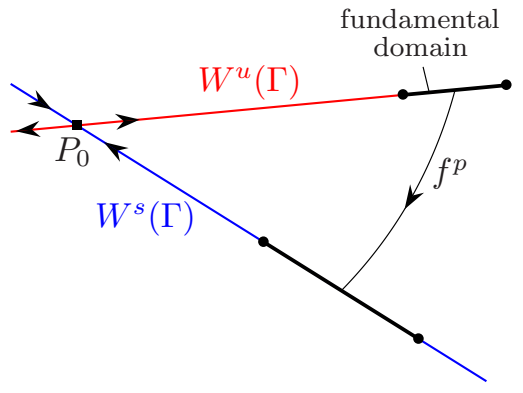

Figure 1: A sketch of the stable (blue) and unstable (red) manifolds of a saddle-type periodic solution $\Gamma$ of a piecewise-linear map $f$ in a neighbourhood of one point of this solution, $P_{0}$. In this neighbourhood the manifolds coincide with the stable and unstable subspaces associated with $P_{0}$. In the simplest instance of a codimension-two subsumed homoclinic connection, a fundamental domain of a branch of the unstable manifold maps linearly to the stable subspace under $p$ iterations of $f$, for some $p \geq 1$. 


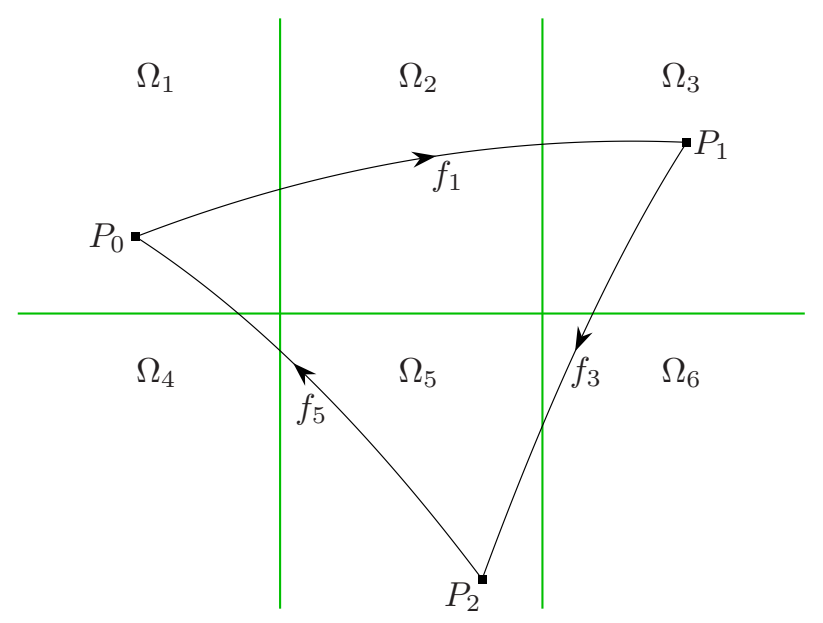

Figure 2: A sketch of the phase space of a map (1.1) that involves six regions showing a 135-cycle, $\left\{P_{0}, P_{1}, P_{2}\right\}$. The green lines represent $\Sigma$.

In two-dimensional parameter space subsumed homoclinic connections typically lie at the intersection of two curves of homoclinic corners. This occurs for a discontinuous map introduced by Mira in [10], and the two-dimensional border-collision normal [16]. In both cases (given as examples below) the intersection is a striking focal point of the overall bifurcation structure. This is because these examples involve $|\lambda \sigma|<1$, and, as explained below by Theorem [3.2, in this case there exists $p \geq 1$ and a sequence of regions $S_{k}$ in which the map has a stable period- $(k n+p)$ solution for all sufficiently large values of $k$. Each $S_{k}$ is triangular, to leading order, and overlaps only $S_{k-1}$ and $S_{k+1}$ for large $k$.

The remainder of the paper is organised as follows. In \$2 we characterise periodic solutions of (1.1) and discuss their existence, admissibility, and stability. Then in $\$ 3$ we carefully set up the codimension-two scenario, assuming $\sigma>1$ for simplicity, and state Theorem 3.2. In $\$ 4$ we provide a proof of Theorem 3.2 and in $\$ 5$ illustrate the result with examples. Finally \$6 provides concluding remarks.

\section{Periodic solutions: notation and basic properties}

We first explain how periodic solutions of (1.1) can be described symbolically. For example, the period-3 solution shown in Fig. 2 is a 135-cycle (cyclic permutations of 135 are also permitted). Ostensibly, 135 is a number, but is termed a 'word' because the indices $1, \ldots, m$ are treated as symbols [8]. A similar exposition involving only two symbols is presented in [17].

We write a word $\mathcal{X}$ of length $n$ as

$$
\mathcal{X}=\mathcal{X}_{0} \cdots \mathcal{X}_{n-1}
$$

where $\mathcal{X}_{i} \in\{1, \ldots, m\}$ for each $i \in\{0, \ldots, n-1\}$. We write $\mathcal{X} \mathcal{Y}$ for the concatenation of two words $\mathcal{X}$ and $\mathcal{Y}$, and $\mathcal{X}^{k}$ for the concatenation of $\mathcal{X}$ with itself $k$ times. 
Given $i \geq 0$, let $f^{i}$ denote the composition of $f$ with itself $i$ times $\left(f^{0}\right.$ is the identity map). Given a word $\mathcal{X}$ of length $n$, let

$$
f_{\mathcal{X}}=f_{\mathcal{X}_{n-1}} \circ \cdots \circ f_{\mathcal{X}_{0}}
$$

denote the composition of the pieces of $f$ in the order determined by $\mathcal{X}$. Let $\Omega_{\mathcal{X}} \subset \Omega$ be the (possibly empty) set of all $P \in \Omega$ for which $f^{i}(P) \in \Omega_{\mathcal{X}_{i}}$ for all $i \in\{0, \ldots, n-1\}$. Roughly speaking, $\Omega_{\mathcal{X}}$ is the set of all points whose forward orbits follow $\mathcal{X}$ initially. Notice $f^{n}(P)=f_{\mathcal{X}}(P)$ for all $P \in \Omega_{\mathcal{X}}$.

Definition 2.1. Given a word $\mathcal{X}$ of length $n$, an $\mathcal{X}$-cycle is an $n$-tuple, $\left\{P_{0}, P_{1}, \ldots, P_{n-1}\right\}$, for which $f_{\mathcal{X}_{0}}\left(P_{0}\right)=P_{1}, f_{\mathcal{X}_{1}}\left(P_{1}\right)=P_{2}, \ldots, f_{\mathcal{X}_{n-1}}\left(P_{n-1}\right)=P_{0}$.

Notice $P_{0}$ in Definition 2.1 is a fixed point of $f_{\mathcal{X}}$. Since $f_{\mathcal{X}}$ is affine, its Jacobian matrix $\mathrm{D} f_{\mathcal{X}}$ is constant in phase space and we have the following result.

Lemma 2.1. A map (1.1) has a unique $\mathcal{X}$-cycle if and only if $I-\mathrm{D} f_{\mathcal{X}}$ is non-singular.

Remark 2.1. Lemma 2.1 implies the $\mathcal{X}$-cycle is completely characterised by the word $\mathcal{X}$, except in the special case that $I-\mathrm{D} f_{\mathcal{X}}$ is singular (which is equivalent to 1 being an eigenvalue of $\left.\mathrm{D} f_{\mathcal{X}}\right)$.

Definition 2.2. The $\mathcal{X}$-cycle of Definition 2.1 is admissible if $P_{0} \in \Omega_{\mathcal{X}}$, and virtual otherwise.

Remark 2.2. Every admissible $\mathcal{X}$-cycle is a periodic solution of $f$. Conversely every periodic solution of $f$ is an admissible $\mathcal{X}$-cycle for some $\mathcal{X}$ that is unique up to cyclic permutation.

If an admissible $\mathcal{X}$-cycle has no points in $\Sigma$ (as in Fig. 2) then some neighbourhood of $P_{0}$ is contained in $\Omega_{\mathcal{X}}$. In this neighbourhood $f^{n}$ is smooth (in fact equal to $f_{\mathcal{X}}$ and so affine). Thus, as with smooth maps [3], the $\mathcal{X}$-cycle is asymptotically stable if both eigenvalues of $\mathrm{D} f_{\mathcal{X}}$ have modulus less than 1 . This corresponds to shaded region shown in Fig. 3 and in fact the converse is true because $f_{\mathcal{X}}$ is affine:

Lemma 2.2. Suppose an admissible $\mathcal{X}$-cycle has no points in $\Sigma$. Let $\tau$ and $\delta$ be the trace and determinant of $\mathrm{D} f_{\mathcal{X}}$. Then the $\mathcal{X}$-cycle is asymptotically stable if and only if $(\tau, \delta)$ belongs to the interior of the triangle shown in Fig. 3 .

\section{Subsumed homoclinic connections and single-round periodic solutions}

In this section we develop a precise symbolic and geometric description of subsumed homoclinic connections building up to the statement of Theorem 3.2. Theorem 3.2 is essence quite simple. It gives, explicitly but just to leading order, the set of all points where the map has a stable single-round periodic solution. However, some technicalities precede the theorem statement because in order for us to provide an explicit formula we need to construct suitable coordinates in parameter space and to prove that single-round periodic solutions are admissible we require certain global assumptions on the nature of the homoclinic connection. 


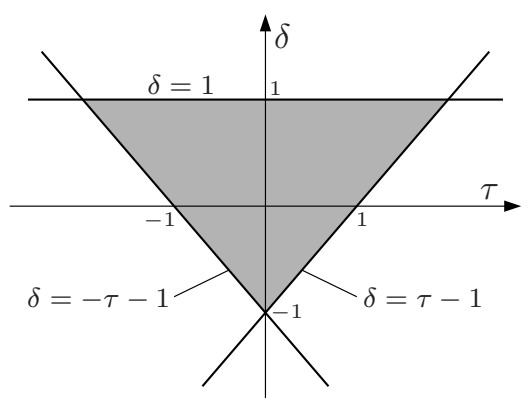

Figure 3: The shaded region is where both eigenvalues of a $2 \times 2$ matrix, with trace $\tau$ and determinant $\delta$, have modulus less than 1 .

\subsection{Persistent properties of the stable and unstable manifolds of an $\mathcal{X}$-cycle}

Let $\Gamma=\left\{P_{0}, P_{1}, \ldots, P_{n-1}\right\}$ be an admissible $\mathcal{X}$-cycle, as in Definition 2.1. The stable manifold of the $\mathcal{X}$-cycle, $W^{s}(\Gamma)$, is defined as all $P \in \Omega \backslash \Gamma$ for which $f^{i}(P)$ converges to the $\mathcal{X}$-cycle as $i \rightarrow \infty$. The unstable manifold of the $\mathcal{X}$-cycle, $W^{u}(\Gamma)$, is defined as all $P \in \Omega \backslash \Gamma$ for which there exists a sequence of preimages $f^{i}(P)$ that converges to the $\mathcal{X}$-cycle as $i \rightarrow-\infty$.

Suppose the $\mathcal{X}$-cycle has no points in $\Sigma$ and the eigenvalues of $\mathrm{D} f_{\mathcal{X}}$, call them $a$ and $b$, satisfy $0<|a|<1<b$. Then $W^{s}(\Gamma)$ and $W^{u}(\Gamma)$ are one-dimensional and emanate linearly from each point $P_{i}$. As they emanate from $P_{0}$, for example, they coincide with the stable and unstable subspaces $E^{s}\left(P_{0}\right)$ and $E^{u}\left(P_{0}\right)$. These are lines through $P_{0}$ with directions given by the eigenvectors of $\mathrm{D} f_{\mathcal{X}}$. Note that $W^{u}(\Gamma)$ has two dynamically independent branches because $b>0$.

We introduce the notation

$$
\mathcal{L}(P, Q)=\{(1-t) P+t Q \mid 0<t<1\}
$$

to denote all points between $P, Q \in \mathbb{R}^{2}$ (if $P \neq Q$ this is a line segment that does not include its endpoints). Observe $P_{0}$ belongs to the interior of $\Omega_{\mathcal{X}}$. Suppose $E^{u}\left(P_{0}\right)$ intersects $\Sigma$ at some $Z \in \Omega$, and $\mathcal{L}\left(P_{0}, Z\right) \subset \Omega_{\mathcal{X}}$, see already Fig. 4. Then every $P \in \mathcal{L}\left(P_{0}, f_{\mathcal{X}}(Z)\right)$ belongs to one branch of $W^{u}(\Gamma)$ because the preimage of $P$ under $f_{\mathcal{X}}$ belongs to $\Omega_{\mathcal{X}}$. For this reason $\{Z\} \cup \mathcal{L}\left(Z, f_{\mathcal{X}}(Z)\right)$ is a fundamental domain for this branch of $W^{u}(\Gamma)$ (it contains exactly one point in every orbit in the branch). This particular fundamental domain is significant because $Z \in \Sigma$ and thus $W^{u}(\Gamma)$ has a kink at $f_{\mathcal{X}}(Z)$ (except in special cases).

\subsection{Subsumed homoclinic connections}

Definition 3.1. The $\mathcal{X}$-cycle is said to have a subsumed homoclinic connection if one branch of $W^{u}(\Gamma)$ is a subset of $W^{s}(\Gamma)$ (or one branch of $W^{s}(\Gamma)$ is a subset of $W^{u}(\Gamma)$ ).

Here we provide sufficient conditions for an $\mathcal{X}$-cycle to have a subsumed homoclinic connection. Let $\mathcal{Y}$ be a word of length $p$. Let $\mathcal{B}$ be a compact, convex set with $P_{0} \in \mathcal{B} \subset \operatorname{int}\left(\Omega_{\mathcal{X}^{2}}\right)$ 
(where int $(\cdot)$ denotes the interior of a set). In Theorem 3.2 we unfold the codimension-two scenario that $Z$ and $f_{\mathcal{X}}(Z)$ both map to $\mathcal{B} \cap E^{s}\left(P_{0}\right)$ under $f_{\mathcal{Y}}$, that is

$$
f_{\mathcal{Y}}(Z), f_{\mathcal{X Y}}(Z) \in \mathcal{B} \cap E^{s}\left(P_{0}\right) .
$$

Since $f_{\mathcal{Y}}$ is affine, (3.1) implies all points in $\mathcal{L}\left(Z, f_{\mathcal{X}}(Z)\right)$ map to $\mathcal{B} \cap E^{s}\left(P_{0}\right)$ under $f_{\mathcal{Y}}$. The assumption that $\mathcal{B} \subset \operatorname{int}\left(\Omega_{\mathcal{X}^{2}}\right)$ is convex ensures $\mathcal{B} \cap E^{s}\left(P_{0}\right)$ is contained in $W^{s}(\Gamma)$ (if $a>0$ the weaker assumption $\mathcal{B} \subset \operatorname{int}\left(\Omega_{\mathcal{X}}\right)$ is sufficient). For (3.1) to imply a subsumed homoclinic connection we need the admissibility assumption

$$
\mathcal{L}\left(Z, f_{\mathcal{X}}(Z)\right) \subset \Omega_{\mathcal{Y}}
$$

and some information about the forward orbit of $Z$.

Proposition 3.1. Suppose (3.1) and (3.2) are satisfied. Suppose either (i) $Z \in \Omega_{\mathcal{Y}}$, or (ii) $Z \in \Omega_{\mathcal{X}}$ and $f_{\mathcal{X}}(Z) \in \Omega_{\mathcal{Y}}$. Then the $\mathcal{X}$-cycle has a subsumed homoclinic connection.

Proof. For any $P \in \mathcal{L}\left(Z, f_{\mathcal{X}}(Z)\right)$ we have $f^{p}(P) \in \mathcal{B} \cap E^{s}\left(P_{0}\right)$ by (3.1) and (3.2) and because $f_{\mathcal{Y}}$ is affine. Under condition (i) we have $f^{p}(Z) \in \mathcal{B} \cap E^{s}\left(P_{0}\right)$, while under condition (ii) we have $f^{n+p}(Z) \in \mathcal{B} \cap E^{s}\left(P_{0}\right)$. In either case every point in the fundamental domain $\{Z\} \cup \mathcal{L}\left(Z, f_{\mathcal{X}}(Z)\right)$ belongs to $W^{s}(\Gamma)$, hence $f$ has a subsumed homoclinic connection.

\subsection{Change of coordinates for variables and parameters}

Now suppose $\Sigma$ and each $f_{j}$ depend smoothly on parameters $\xi, \zeta \in \mathbb{R}$. Theorem 3.2 includes the following assumption.

Assumption 3.2. At $(\xi, \zeta)=(0,0)$,

i) (1.1) has an admissible $\mathcal{X}$-cycle with no points on $\Sigma$ and its eigenvalues satisfy $0<$ $|a|<1<b$

ii) $Z$, defined as above, lies at a transverse intersection between $E^{u}\left(P_{0}\right)$ and $\Sigma$, and

iii) $\mathcal{L}\left(P_{0}, Z\right) \subset \operatorname{int}\left(\Omega_{\mathcal{X}}\right)$ and $\mathcal{L}\left(Z, f_{\mathcal{X}}(Z)\right) \subset \operatorname{int}\left(\Omega_{\mathcal{Y}}\right)$.

Since the $\mathcal{X}$-cycle is hyperbolic it persists smoothly as a saddle with no points on $\Sigma$ in a neighbourhood of $(\xi, \zeta)=(0,0)$. Its eigenvalues $a$ and $b$ vary smoothly with $\xi$ and $\zeta$ as does $Z$ because transverse intersections persist.

Let $q_{\text {unstab }}=Z-P_{0}$. This is an eigenvector of $\mathrm{D} f_{\mathcal{X}}$ corresponding to the eigenvalue $b$. Let $q_{\text {stab }}$ be a smoothly varying eigenvector of $\mathrm{D} f_{\mathcal{X}}$ corresponding to the eigenvalue $a$. Form the matrix $T=\left[\begin{array}{ll}q_{\text {stab }} & q_{\text {unstab }}\end{array}\right]$. Then

$$
h(P)=T^{-1}\left(P-P_{0}\right)
$$

gives the coordinates of any $P \in \Omega$ relative to the eigenvectors. The function $h=\left(h_{1}, h_{2}\right)$ provides a coordinate change on the variables of (1.1) that we use in $\$ 4$. 


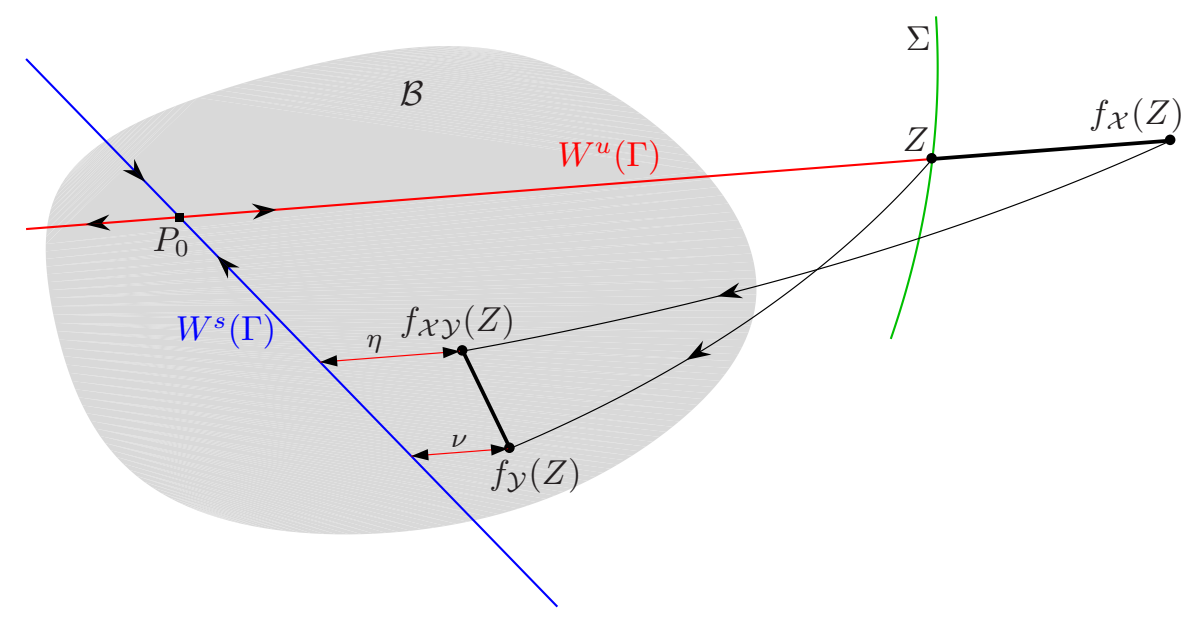

Figure 4: A sketch of the stable (blue) and unstable (red) manifolds of the $\mathcal{X}$-cycle near $P_{0}$. Here the manifolds coincide with the lines $E^{s}\left(P_{0}\right)$ and $E^{u}\left(P_{0}\right)$. The meaning of $\eta$ and $\nu$, defined by (3.4) $-(3.5)$, is indicated.

Let

$$
\begin{aligned}
& \eta=h_{2}\left(f_{\mathcal{X Y}}(Z)\right), \\
& \nu=h_{2}\left(f_{\mathcal{Y}}(Z)\right) .
\end{aligned}
$$

As illustrated in Fig. 目, these values provide a measure for the distance of $f_{\mathcal{X Y}}(Z)$ and $f_{\mathcal{Y}}(Z)$ from $E^{s}\left(P_{0}\right)$ in the direction $q_{\text {unstab }}$.

Theorem 3.2 assumes the codimension-two condition (3.1) holds at $(\xi, \zeta)=(0,0)$. This condition implies $(\eta, \nu)=(0,0)$. In order for $\xi$ and $\zeta$ to unfold the codimension-two point in a generic fashion we require $\left.\operatorname{det}(J)\right|_{(\xi, \zeta)=(0,0)} \neq 0$, where

$$
J=\left[\begin{array}{ll}
\frac{\partial \eta}{\partial \xi} & \frac{\partial \eta}{\partial \zeta} \\
\frac{\partial \nu}{\partial \xi} & \frac{\partial \nu}{\partial \zeta}
\end{array}\right] .
$$

This ensures that the coordinate change on the parameters, $(\xi, \zeta) \rightarrow(\eta, \nu)$, is locally invertible, and we write this coordinate change as

$$
(\eta, \nu)=\psi(\xi, \zeta)
$$

\subsection{Main result}

An $\mathcal{X}^{k} \mathcal{Y}$-cycle, which we write as $\left\{\hat{P}_{0}^{(k)}, \ldots, \hat{P}_{k n+p-1}^{(k)}\right\}$, has period $k n+p$ and is a single-round periodic solution in the sense that it involves a single 'excursion' following the word $\mathcal{Y}$. We centre our analysis around the particular point $\hat{P}_{k n}^{(k)}$ which, roughly speaking, is the point of the $\mathcal{X}^{k} \mathcal{Y}$-cycle that gets mapped under $f_{\mathcal{Y}}$. 
Definition 3.3. At $(\xi, \zeta)=(0,0)$ let $\mathcal{M}$ be a neighbourhood of $\mathcal{L}\left(Z, f_{\mathcal{X}}(Z)\right)$. For each $k \geq 0$, let $S_{k} \subset \mathbb{R}^{2}$ be the set of all $(\xi, \zeta)$ for which (1.1) has an admissible, asymptotically stable $\mathcal{X}^{k} \mathcal{Y}$-cycle with $\hat{P}_{k n}^{(k)} \in \mathcal{M}$.

In Theorem 3.2, $\lambda=a(0,0)$ and $\sigma=b(0,0)$ denote the eigenvalues of the $\mathcal{X}$-cycle at $(\xi, \zeta)=(0,0)$. Given $k \geq 0$ and $\gamma \geq 0$, let

$$
\Delta_{k, \gamma}^{ \pm}=\left\{(\eta, \nu) \mid \eta<\sigma^{-(k-1)} \pm \gamma^{k}, \nu>\sigma^{-k} \mp \gamma^{k}, \nu<\eta+(\sigma-1) \sigma^{-k} \pm \gamma^{k}\right\},
$$

shown in Fig. 5 .

Theorem 3.2. Suppose Assumption 3.2 is satisfied, (3.1) is satisfied at $(\xi, \zeta)=(0,0)$, and $\mathcal{X}_{0} \neq \mathcal{Y}_{0}$. Let $\lambda=a(0,0)$ and $\sigma=b(0,0)$, suppose $|\lambda| \sigma<1$, and let $\gamma=\max \left[\sigma^{-\frac{3}{2}},|\lambda|^{\frac{1}{2}} \sigma^{-\frac{1}{2}}\right]$. Also suppose $\operatorname{det}(J) \neq 0$. Then there exists $k_{\min } \geq 0$, a neighbourhood $\mathcal{M}$ as in Definition 3.3, and a neighbourhood $\mathcal{N}$ of $(\xi, \zeta)=(0,0)$, such that in $\mathcal{N}$

$$
\psi^{-1}\left(\Delta_{k, \gamma}^{-}\right) \subset S_{k} \subset \psi^{-1}\left(\Delta_{k, \gamma}^{+}\right),
$$

for all $k \geq k_{\min }$.

Theorem 3.2, proved in \&4, provides inner and outer bounds for $S_{k}$. Since $\gamma^{k} \rightarrow 0$ faster than $\sigma^{-k} \rightarrow 0$, the relative error that this provides for the location of $S_{k}$ goes to zero as $k \rightarrow \infty$, specifically $\frac{\operatorname{Area}\left(\Delta_{k, \gamma}^{+} \backslash \Delta_{k, \gamma}^{-}\right)}{\operatorname{Area}\left(\Delta_{k, \gamma}^{+}\right)} \rightarrow 0$.

We conclude this section with some technical remarks and determine how the $S_{k}$ overlap one another, Fig. 6. Overlaps are significant as they imply two stable periodic solutions coexist.

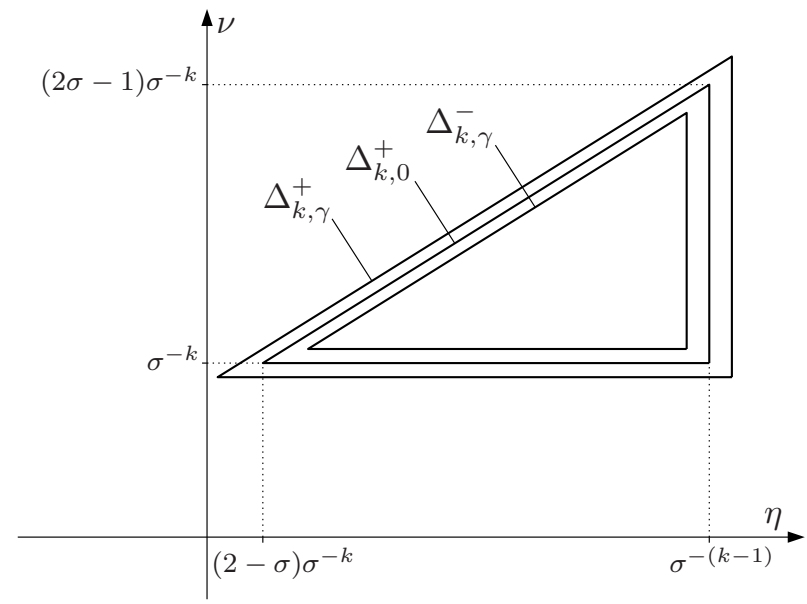

Figure 5: The regions $\Delta_{k, \gamma}^{-}$and $\Delta_{k, \gamma}^{+}$provide inner and outer bounds for $\psi\left(S_{k}\right)$, where $\psi\left(S_{k}\right)$ represents pairs $(\eta, \nu)$ for which (1.1) has an admissible, stable $\mathcal{X}^{k} \mathcal{Y}$-cycle, see (3.7) and Definition 3.3. The leading order (large $k$ ) approximation to $\psi\left(S_{k}\right)$ is $\Delta_{k, 0}^{+}=\Delta_{k, 0}^{-}$because we assume $\gamma<\frac{1}{\sigma}$ (Theorem 3.2 provides a specific value for $\gamma$ ). 
Remark 3.1. For each of the examples in 95 the $S_{k}$ involve three smooth boundaries. To prove that this is the case in general, which has not been achieved by Theorem 3.2, we must precisely characterise the bifurcations to which each boundary corresponds. While the boundary approximated by $\nu=\eta+(\sigma-1) \sigma^{-k}$ is always where the $\mathcal{X}^{k} \mathcal{Y}$-cycle loses stability by attaining an eigenvalue of -1 , a characterisation of the other boundaries depends on whether $f$ is discontinuous or continuous. In both cases these boundaries are border-collision bifurcations where one point of the $\mathcal{X}^{k} \mathcal{Y}$-cycle collides with $\Sigma$. If $f$ is discontinuous, these collisions occur near $Z$. If $f$ is continuous, $W^{u}(\Gamma)$ is continuous as so must have at least one other intersection with $\Sigma$ [19]. In this case one of the two collisions occurs near this other intersection in accordance with the unfolding of a shrinking point [17, 18].

Remark 3.2. The assumption $\mathcal{X}_{0} \neq \mathcal{Y}_{0}$ is a natural consequence of $Z \in \Sigma$ and allows us to prove $S_{k} \subset \psi^{-1}\left(\Delta_{k, \gamma}^{+}\right)$in a simple way. In practise we can always impose $\mathcal{X}_{0} \neq \mathcal{Y}_{0}$ by choosing an appropriate cyclic permutation of $\mathcal{X}$. Similarly the assumption $\hat{P}_{k n}^{(k)} \in \mathcal{M}$ ensures $S_{k}$ does not include other points near $(\xi, \zeta)=(0,0)$ due to a peculiar global arrangement of the regions $\Omega_{\mathcal{X}}$ and $\Omega_{\mathcal{Y}}$.

Remark 3.3. The assumption $\mathcal{L}\left(Z, f_{\mathcal{X}}(Z)\right) \subset \operatorname{int}\left(\Omega_{\mathcal{Y}}\right)$ is relatively strong as it refers to almost all orbits in the homoclinic connection. This 'global' assumption is used to ensure that the $\mathcal{X}^{k} \mathcal{Y}$-cycles are admissible and was circumvented for a similar situation in [19] by having detailed knowledge of one homoclinic orbit and its relation to $\Sigma$.

Remark 3.4. The leading order terms in our large $k$ asymptotic expansions (see \&4) are $\mathcal{O}\left(\sigma^{-k}\right)$ (this is big-O notation). Second order terms are either $\mathcal{O}\left(\lambda^{k}\right)$ or $\mathcal{O}\left(\sigma^{-2 k}\right)$. We have designed $\gamma$ so that $\gamma^{k}$ is smaller than leading order (so that the relative error in $S_{k}$ decreases with $k$ ) but bigger than second order (so that higher order terms can be ignored).

Proposition 3.3. Let $j \geq 1$. If the conditions of Theorem 3.2 hold then, for sufficiently large values of $k, S_{k} \cap S_{k+j}=\varnothing$ if and only if $j>1$.

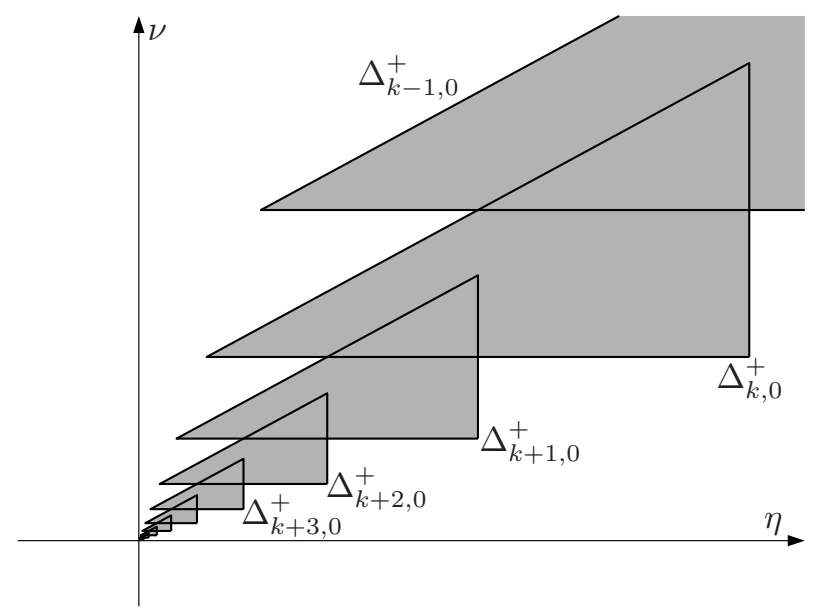

Figure 6: A sketch of $\Delta_{k+j, 0}^{+}=\Delta_{k+j, 0}^{-}$(the leading order approximation to $\psi\left(S_{k+j}\right)$ ) for several values of $j$. 
Proof. The top-right corner of $\Delta_{k+1,0}^{+}$is $(\eta, \nu)=\left(\sigma^{-k}, \frac{2 \sigma-1}{\sigma} \sigma^{-k}\right)$. By checking all three inequalities in (3.8) it can easily be seen that this corner belongs to $\operatorname{int}\left(\Delta_{k, 0}^{+}\right)$because $\sigma>1$. Thus $\operatorname{int}\left(\Delta_{k, 0}^{+}\right) \cap \Delta_{k+1,0}^{+} \neq \varnothing$, and so $S_{k} \cap S_{k+1} \neq \varnothing$ (using Theorem 3.2) for sufficiently large values of $k$ because $\gamma^{k} \rightarrow 0$ faster than $\sigma^{-k} \rightarrow 0$.

The top-right corner of $\Delta_{k+2,0}^{+}$is $(\eta, \nu)=\left(\sigma^{-(k+1)}, \frac{2 \sigma-1}{\sigma^{2}} \sigma^{-k}\right)$ and lies below the bottom edge of $\Delta_{k, 0}^{+}$because $\frac{2 \sigma-1}{\sigma^{2}}=1-\frac{(\sigma-1)^{2}}{\sigma^{2}}<1$. This is also the case for any $\Delta_{k+j, 0}^{+}$with $j>2$. Thus, for all $j>1$, we have $\Delta_{k, 0}^{+} \cap \Delta_{k+j, 0}^{+}=\varnothing$ and so $S_{k} \cap S_{k+j} \neq \varnothing$ (using Theorem 3.2) for sufficiently large values of $k$.

\section{Proof of Theorem 3.2}

The $\mathcal{X}$-cycle exists, is unique, and has no points on $\Sigma$ in some compact neighbourhood $\mathcal{N}$ of $(\xi, \zeta)=(0,0)$. We can also assume that in $\mathcal{N}$ the eigenvalues satisfy $0<|a|<1<b$, that $Z$ is well-defined and varies smoothly, and that $h$ and $\psi$ are well-defined and invertible.

\subsection{Change of variables}

We write the change of variables (3.3) as $(u, v)=h(x, y)$. For any word $\mathcal{W}$, let $g_{\mathcal{W}}=$ $h \circ f_{\mathcal{W}} \circ h^{-1}$ denote $f_{\mathcal{W}}$ in $(u, v)$-coordinates, and write $g_{\mathcal{W}}=\left(g_{\mathcal{W}, 1}, g_{\mathcal{W}, 2}\right)$. Then

$$
g_{\mathcal{X}}(x, y)=\left[\begin{array}{l}
a u \\
b v
\end{array}\right]
$$

and write

$$
g_{\mathcal{Y}}(u, v)=\left[\begin{array}{l}
c_{11} u+c_{12} v+c_{13} \\
c_{21} u+c_{22} v+c_{23}
\end{array}\right],
$$

for some parameter-dependent coefficients $c_{11}, \ldots, c_{23} \in \mathbb{R}$.

By the definition of $q_{\text {unstab }}$, we have $h(Z)=(0,1)$ and $h\left(f_{\mathcal{X}}(Z)\right)=(0, b)$. Then (3.4) and (3.5) imply $\eta=g_{\mathcal{Y}, 2}(0, b)$ and $\nu=g_{\mathcal{Y}, 2}(0,1)$, see Fig. (7), and so

$$
\begin{aligned}
& c_{22}=\frac{\eta-\nu}{b-1}, \\
& c_{23}=\frac{-\eta+b \nu}{b-1} .
\end{aligned}
$$

Formulas for the other coefficients in (4.2) will not be needed.

\section{$4.2 \mathcal{X}^{k} \mathcal{Y}$-cycles}

By composing (4.1) and (4.2) we obtain

$$
g_{\mathcal{X}^{k} \mathcal{Y}}(u, v)=\left[\begin{array}{l}
a^{k} c_{11} u+b^{k} c_{12} v+c_{13} \\
a^{k} c_{21} u+b^{k} c_{22} v+c_{23}
\end{array}\right] .
$$


The trace and determinant of $\mathrm{D} g_{\mathcal{X}^{k} \mathcal{Y}}$ are therefore

$$
\begin{aligned}
& \tau_{k}=a^{k} c_{11}+b^{k} c_{22}, \\
& \delta_{k}=a^{k} b^{k}\left(c_{11} c_{22}-c_{12} c_{21}\right),
\end{aligned}
$$

respectively. By Lemma 2.1, there exists a unique $\mathcal{X}^{k} \mathcal{Y}$-cycle if $\operatorname{det}\left(I-\mathrm{D} f_{\mathcal{X}^{k} \mathcal{Y}}\right) \neq 0$. This is equivalent to $\delta_{k}-\tau_{k}+1 \neq 0$, because $\mathrm{D} f_{\mathcal{X}^{k} \mathcal{Y}}$ and $\mathrm{D} g_{\mathcal{X}^{k} \mathcal{Y}}$ are similar and $\operatorname{det}\left(I-\mathrm{D} g_{\mathcal{X}^{k}}\right)=$ $\delta_{k}-\tau_{k}+1$.

For each $i$, let $Q_{i}=h\left(\hat{P}_{i}^{(k)}\right)$ (in $Q_{i}$ the $k$-dependence is suppressed for brevity). Notice $Q_{0}$ is a fixed point of $g_{\mathcal{X}^{k} \mathcal{Y}}$. By calculating this point via (4.5) and iterating it $k$ times under $g_{\mathcal{X}}$ we obtain

$$
Q_{k n}=\frac{1}{\delta_{k}-\tau_{k}+1}\left[\begin{array}{l}
a^{k} c_{13}-a^{k} b^{k}\left(c_{22} c_{13}-c_{12} c_{23}\right) \\
b^{k} c_{23}+a^{k} b^{k}\left(c_{21} c_{13}-c_{11} c_{23}\right)
\end{array}\right],
$$

assuming $\delta_{k}-\tau_{k}+1 \neq 0$. We also write $Q_{k n}=\left(u^{(k)}, v^{(k)}\right)$.

\subsection{Accommodating relatively large values of $\eta$ and $\nu$}

In (4.6) -(4.8), the largest terms are $b^{k} c_{22}$ in (4.6) and $b^{k} c_{23}$ in (4.8) because $b>1$ and $k$ is large. But in $S_{k}$ we must have $\left|\tau_{k}\right|<2$ (for stability) and $v^{(k)}$ be not too large (for admissibility). Intuitively, (4.3)-(4.4) imply that $\eta$ and $\nu$ need to be $\mathcal{O}\left(\sigma^{-k}\right)$ throughout $S_{k}$. Here we establish this formally (although details are omitted for brevity).

We partition $\psi(\mathcal{N})$ into three components using some values $R_{1}, R_{2}>0$ as follows. Let $\mathcal{N}_{1}$ be all $(\eta, \nu) \in \psi(\mathcal{N})$ for which $|\eta-\nu|>R_{1} \sigma^{-k}$, let $\mathcal{N}_{2}$ be all $(\eta, \nu) \in \psi(\mathcal{N})$ for which $|\eta-\nu| \leq R_{1} \sigma^{-k}$ and $|\eta|>R_{2} \sigma^{-k}$, and let $\mathcal{N}_{3}$ be all other points in $\psi(\mathcal{N})$. From (4.3) and

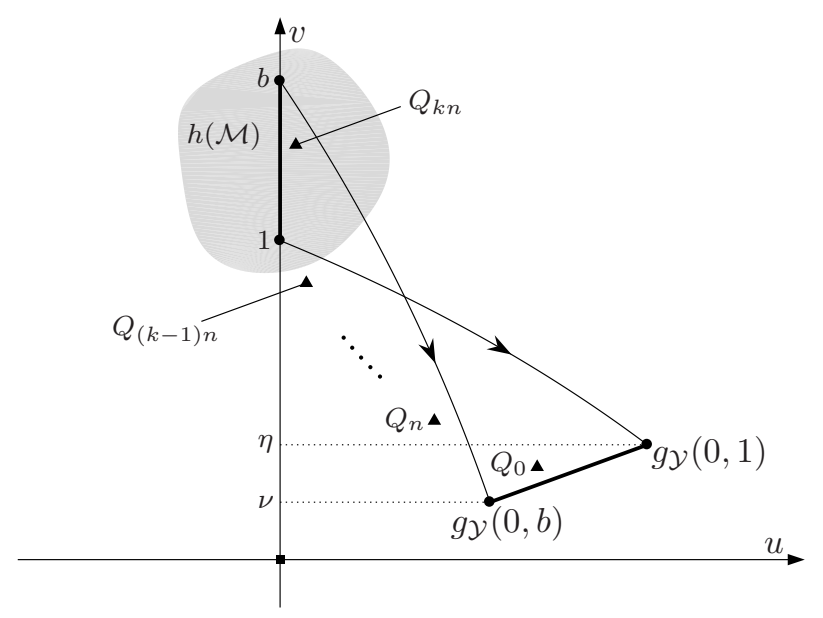

Figure 7: Phase space in $(u, v)$-coordinates, where $(u, v)=h(x, y)$, near a subsumed homoclinic connection. Some points of an $\mathcal{X}^{k} \mathcal{Y}$-cycle are shown. For each $j \in\{0, \ldots, k-1\}, Q_{j n}$ maps to $Q_{(j+1) n}$ under $g_{\mathcal{X}}$, while $Q_{k n}$ maps to $Q_{0}$ under $g_{\mathcal{Y}}$. Notice $h(\mathcal{M})$ is a neighbourhood of the interval $(1, b)$ of the $v$-axis. 
(4.6) we can find $R_{1}>0$ such that throughout $\mathcal{N}_{1}$ we have $\left|\tau_{k}\right|>2$, for all sufficiently large values of $k$, and so, if it exists, the $\mathcal{X}^{k} \mathcal{Y}$-cycle is unstable. Such an $R_{1}$ can be constructed from values $b_{\min }, b_{\max }>1$ for which $b_{\min } \leq b \leq b_{\max }$ throughout $\psi(\mathcal{N})$. Similarly we can find $R_{2}>0$ such that throughout $\mathcal{N}_{2}$ we have $\left|v^{(k)}\right|>b+1$, say, for all sufficiently large values of $k$, and so $Q_{k n} \notin h(\mathcal{M})$, assuming $\mathcal{M}$ is sufficiently small.

Consequently it remains for us to consider $(\eta, \nu) \in \mathcal{N}_{3}$ for which we may treat $\tilde{\eta}=\sigma^{k} \eta$ and $\tilde{\nu}=\sigma^{k} \nu$ as order-1 quantities.

\subsection{Existence}

From (4.3) and (4.6)-(4.7) we have

$$
\begin{aligned}
\tau_{k} & =\frac{\tilde{\eta}-\tilde{\nu}}{\sigma-1}+\mathcal{O}\left(\sigma^{-k}\right), \\
\delta_{k} & =\mathcal{O}\left(\lambda^{k} \sigma^{k}\right),
\end{aligned}
$$

and so

$$
\delta_{k}-\tau_{k}+1=1-\frac{\tilde{\eta}-\tilde{\nu}}{\sigma-1}+\mathcal{O}\left(\sigma^{-k}\right)+\mathcal{O}\left(\lambda^{k} \sigma^{k}\right) .
$$

By substituting $\tilde{\nu}>\tilde{\eta}-\sigma+1+2 \gamma^{k} \sigma^{k}$ (which holds for all points in $\Delta_{k, \gamma}^{-}$) into (4.9), we

obtain $\delta_{k}-\tau_{k}+1>\frac{2 \sigma^{k} \gamma^{k}}{\sigma-1}+\mathcal{O}\left(\sigma^{-k}\right)+\mathcal{O}\left(\lambda^{k} \sigma^{k}\right)$, which is positive for sufficiently large values of $k$. Thus the $\mathcal{X}^{k} \mathcal{Y}$-cycle exists and is unique in $\Delta_{k, \gamma}^{-}$(for large $k$ ).

Similarly by substituting $\tilde{\nu}<\tilde{\eta}-\sigma+1-2 \gamma^{k} \sigma^{k}$ into (4.9) we obtain $\delta_{k}-\tau_{k}+1<0$, for large $k$, and so here the $\mathcal{X}^{k} \mathcal{Y}$-cycle cannot be stable.

\subsection{Stability}

By substituting $\tilde{\nu}<\tilde{\eta}+\sigma-1-\gamma^{k} \sigma^{k}$ (i.e. below the angled edge of $\Delta_{k, \gamma}^{-}$) into

$$
\delta_{k}+\tau_{k}+1=1+\frac{\tilde{\eta}-\tilde{\nu}}{\sigma-1}+\mathcal{O}\left(\sigma^{-k}\right)+\mathcal{O}\left(\lambda^{k} \sigma^{k}\right)
$$

we obtain $\delta_{k}+\tau_{k}+1>\frac{\sigma^{k} \gamma^{k}}{\sigma-1}+\mathcal{O}\left(\sigma^{-k}\right)+\mathcal{O}\left(\lambda^{k} \sigma^{k}\right)$, which is positive for sufficiently large values of $k$. Since also $\delta_{k} \rightarrow 0$ as $k \rightarrow \infty$, we conclude that in $\Delta_{k, \gamma}^{-}$the pair $\left(\tau_{k}, \delta_{k}\right)$ belongs to the triangle of Fig. 3 and so if the $\mathcal{X}^{k} \mathcal{Y}$-cycle is admissible then it is also stable.

By similarly substituting $\tilde{\nu}>\tilde{\eta}+\sigma-1+\gamma^{k} \sigma^{k}$ into (4.10) we obtain $\delta_{k}+\tau_{k}+1<0$, for large $k$, and so here the $\mathcal{X}^{k} \mathcal{Y}$-cycle cannot be stable.

\subsection{Admissibility}

From (4.4) and (4.8) we have $u^{(k)}=\mathcal{O}\left(\lambda^{k}\right)$ and

$$
v^{(k)}=v^{(\infty)}+\mathcal{O}\left(\sigma^{-k}\right)+\mathcal{O}\left(\lambda^{k} \sigma^{k}\right),
$$


where

$$
v^{(\infty)}=\frac{-\tilde{\eta}+\sigma \tilde{\nu}}{\sigma-1-\tilde{\eta}+\tilde{\nu}} .
$$

If $\tilde{\eta}$ and $\tilde{\nu}$ are such that $1<v^{(\infty)}<\sigma$, then Assumption 3.2 implies $Q_{k n} \in \Omega_{\mathcal{Y}}$ (also $Q_{k n} \in h(\mathcal{M})$ ) for large values of $k$. Also for each $j=0, \ldots, k-1$ the point $Q_{j n}$ is in $\mathcal{B}$ or close to $\mathcal{L}\left(P_{0}, Z\right)$, for large values of $k$, and so $Q_{j n} \in \Omega_{\mathcal{X}}$. In this case the $\mathcal{X}^{k} \mathcal{Y}$-cycle is admissible and $(\eta, \nu) \in \psi\left(S_{k}\right)$.

If instead $v^{(\infty)}<1$ then for sufficiently large values of $k$ either $Q_{k n} \in \Omega_{\mathcal{X}}$ (in which case the $\mathcal{X}^{k} \mathcal{Y}$-cycle is virtual because $\left.\mathcal{X}_{0} \neq \mathcal{Y}_{0}\right)$ or $Q_{k n} \notin h(\mathcal{M})$. In either case $(\eta, \nu) \notin \psi\left(S_{k}\right)$. If $v^{(\infty)}>\sigma$, then either $Q_{(k-1) n} \in \Omega_{y}$ or $Q_{k n} \notin h(\mathcal{M})$ and we reach the same conclusion.

Simply solving $v^{(\infty)}=1$ and $v^{(\infty)}=\sigma$ yields $\tilde{\nu}=1$ and $\tilde{\eta}=\sigma$, respectively, and this provides a rough explanation for the vertical and horizontal edges of the $\Delta_{k, \gamma}^{ \pm}$. More formally it is a simple exercise to use the formula (4.12) to show that in $\Delta_{k, \gamma}^{-}$we have

$$
1+\frac{1}{2} \beta^{k} \sigma^{k}+\mathcal{O}\left(\sigma^{-k}\right)+\mathcal{O}\left(\lambda^{k} \sigma^{k}\right)<v^{(\infty)}<\sigma-\frac{1}{2} \beta^{k} \sigma^{k}+\mathcal{O}\left(\sigma^{-k}\right)+\mathcal{O}\left(\lambda^{k} \sigma^{k}\right),
$$

and so in $\Delta_{k, \gamma}^{-}$the $\mathcal{X}^{k} \mathcal{Y}$ is admissible with $Q_{k n} \in h(\mathcal{M})$ for sufficiently large values of $k$. Also if $\tilde{\nu} \geq \tilde{\eta}-\sigma+1-2 \gamma^{k} \sigma^{k}$ and $\tilde{\nu} \leq \tilde{\eta}+\sigma-1+\gamma^{k} \sigma^{k}$ (above it was shown that these are necessary for the $\mathcal{X}^{k} \mathcal{Y}$-cycle to be stable), then outside $\Delta_{k, \gamma}^{+}$we have either $v^{(\infty)}<$ $1-\frac{1}{2} \beta^{k} \sigma^{k}+\mathcal{O}\left(\sigma^{-k}\right)+\mathcal{O}\left(\lambda^{k} \sigma^{k}\right)$ or $v^{(\infty)}>\sigma+\frac{1}{2} \beta^{k} \sigma^{k}+\mathcal{O}\left(\sigma^{-k}\right)+\mathcal{O}\left(\lambda^{k} \sigma^{k}\right)$ and so the $\mathcal{X}^{k} \mathcal{Y}$-cycle is either virtual or $Q_{k n} \notin h(\mathcal{M})$.

\section{$5 \quad$ Examples}

\subsection{A discontinuous map}

Here we consider the discontinuous map of [10]:

$$
f(x, y)= \begin{cases}f_{1}(x, y), & x<0 \\ f_{2}(x, y), & x \geq 0\end{cases}
$$

where

$$
\begin{aligned}
& f_{1}(x, y)=\left[\begin{array}{c}
\bar{\lambda}_{1} x+y+\bar{a} \\
\bar{\beta} x
\end{array}\right], \\
& f_{2}(x, y)=\left[\begin{array}{c}
\bar{\lambda}_{2} x+y+\bar{b} \\
\bar{\beta} x
\end{array}\right] .
\end{aligned}
$$

Bars have been added to the parameters to avoid confusion with the notation that has already been developed. As in Figure 7 of [10] we fix

$$
\bar{b}=-2, \quad \bar{\lambda}_{2}=1.5, \quad \bar{\beta}=0.3,
$$

and vary the remaining two parameters. 
Fig. 8 shows a two-parameter bifurcation diagram. Regions where there exists a stable periodic solution (up to period 30) are coloured by period. The two white lines are curves of codimension-one homoclinic corners. The intersection of these curves is a codimension-two point at which a fixed point $P_{0}$ in $x>0$ has eigenvalues $\lambda \approx-0.18$ and $\sigma \approx 1.68$ and a subsumed homoclinic connection. Fig. 9 shows a phase portrait at sample parameter values near the codimension-two point.

The codimension-two point satisfies the conditions of Theorem 3.2 . Here $\mathcal{X}=2$ and $\mathcal{Y}=1$. The point $Z$ lies at a transverse intersection between $E^{u}\left(P_{0}\right)$ and the switching manifold $x=0$, and condition (iii) of Assumption 3.2 can be verified by inspection. Also $\operatorname{det}(J) \neq 0$ is a consequence of the transversal intersection between the curves of homoclinic corners.

The regions $S_{k}$ predicted by Theorem 3.2 are labelled in Fig. 8 up to $k=6$ and visible for some larger values of $k$ (other coloured regions correspond to multi-round periodic solutions). The phase portrait in Fig. 9 corresponds to parameter values in $S_{5} \cap S_{6}$ and the corresponding stable $2^{5} 1$ and $2^{6} 1$-cycles are plotted.

\subsection{The border-collision normal form}

The continuous map

$$
f(x, y)= \begin{cases}f_{1}(x, y), & x \leq 0 \\ f_{2}(x, y), & x \geq 0\end{cases}
$$

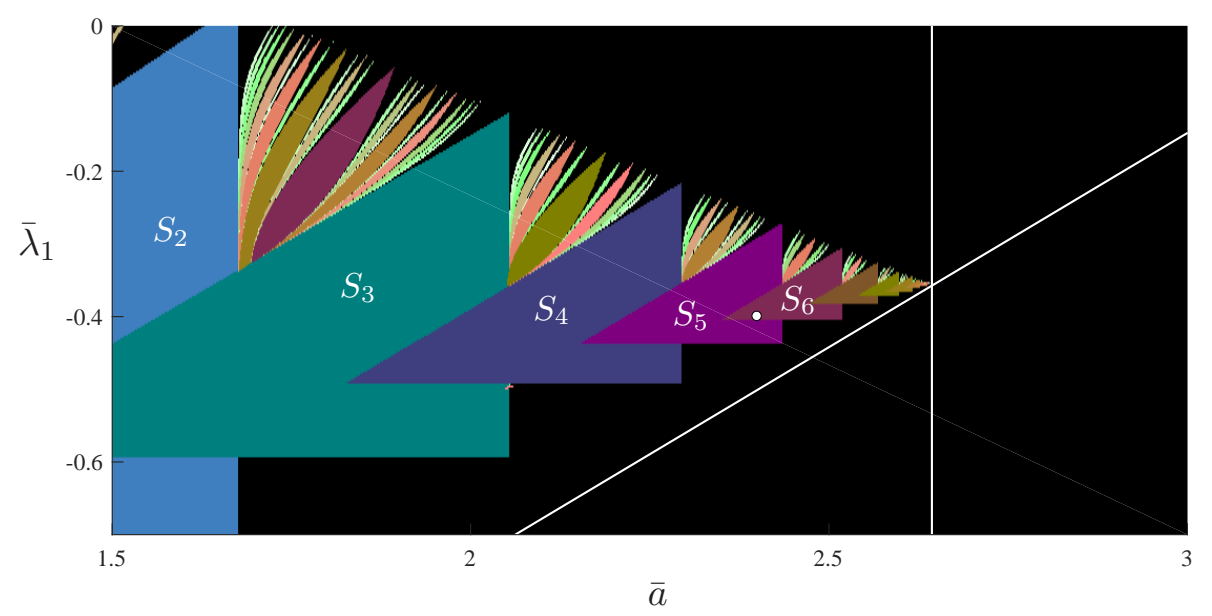

Figure 8: Regions where the discontinuous map (5.1) with (5.3) has a stable periodic solution (different colours correspond to different periods). This was computed numerically via a brute-force search on a $1024 \times 256$ grid of $\left(\bar{a}, \bar{\lambda}_{1}\right)$-values up to period 30 . At points where multiple stable periodic solutions were found the highest period is indicated. The white lines are curves of homoclinic corners that intersect at a subsumed homoclinic connection. The white circle indicates the parameter values of Fig. 9. 


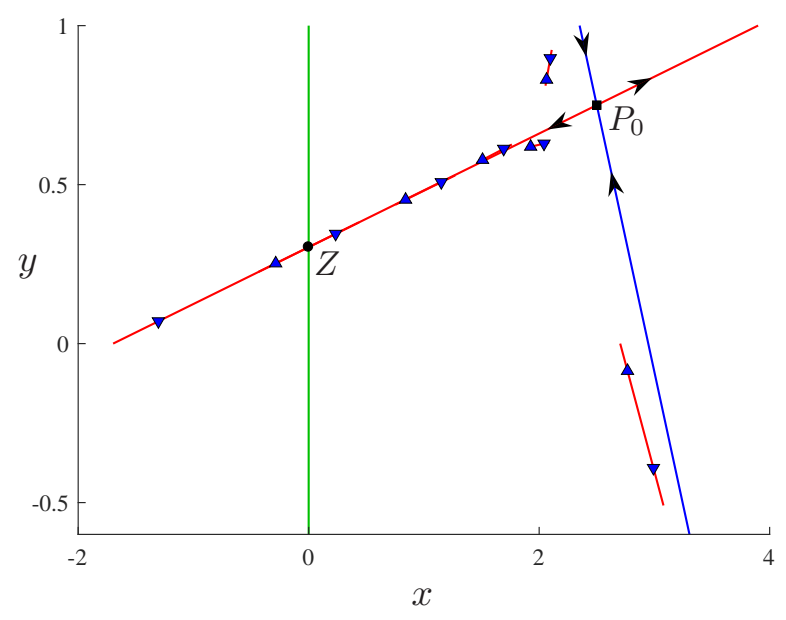

Figure 9: A phase portrait of the discontinuous map (5.1) with (5.3) and $\left(\bar{a}, \bar{\lambda}_{1}\right)=$ $(2.4,-0.4)$. Parts of the stable and unstable manifolds of the fixed point $P_{0}$ are coloured blue and red, respectively. The intersection $Z$ (defined in 93 ) of one branch of $W^{u}\left(P_{0}\right)$ with $\Sigma(x=0)$ is indicated. The stable $2^{5} 1$ and $2^{6} 1$-cycles are shown with two sets of triangles of different orientations.

where

$$
\begin{aligned}
& f_{1}(x, y)=\left[\begin{array}{c}
\tau_{1} x+y+1 \\
-\delta_{1} x
\end{array}\right], \\
& f_{2}(x, y)=\left[\begin{array}{c}
\tau_{2} x+y+1 \\
-\delta_{2} x
\end{array}\right] .
\end{aligned}
$$

is known as the two-dimensional border-collision normal form (except the border-collision bifurcation parameter, usually called $\mu$, has been scaled to 1) [11, 15]. Fig. 10 shows a two-parameter bifurcation diagram using

$$
\tau_{1}=2, \quad \delta_{1}=0.75 .
$$

Four distinct curves of homoclinic corners emanate from a common intersection point at $\left(\delta_{2}, \tau_{2}\right)=(1.5,-0.5)$. Here a fixed point $P_{0}$ in $x<0$ has eigenvalues $\lambda=0.5$ and $\sigma=1.5$ and a subsumed homoclinic connection. The conditions of Theorem 3.2 can be readily verified with $\mathcal{X}=1$ and $\mathcal{Y}=22$, and the regions $S_{k}$ are shown in Fig. 10. Fig. 11] shows a phase portrait at parameter values relatively near the codimension-two point.

\subsection{An example with $n>1$}

Finally we provide an example for which the $\mathcal{X}$-cycle is not a fixed point. Fig. 12 shows a different two-parameter slice of the four-dimensional parameter space of the border-collision normal form (5.4). Specifically we fix

$$
\tau_{2}=-2.5, \quad \delta_{2}=2 .
$$




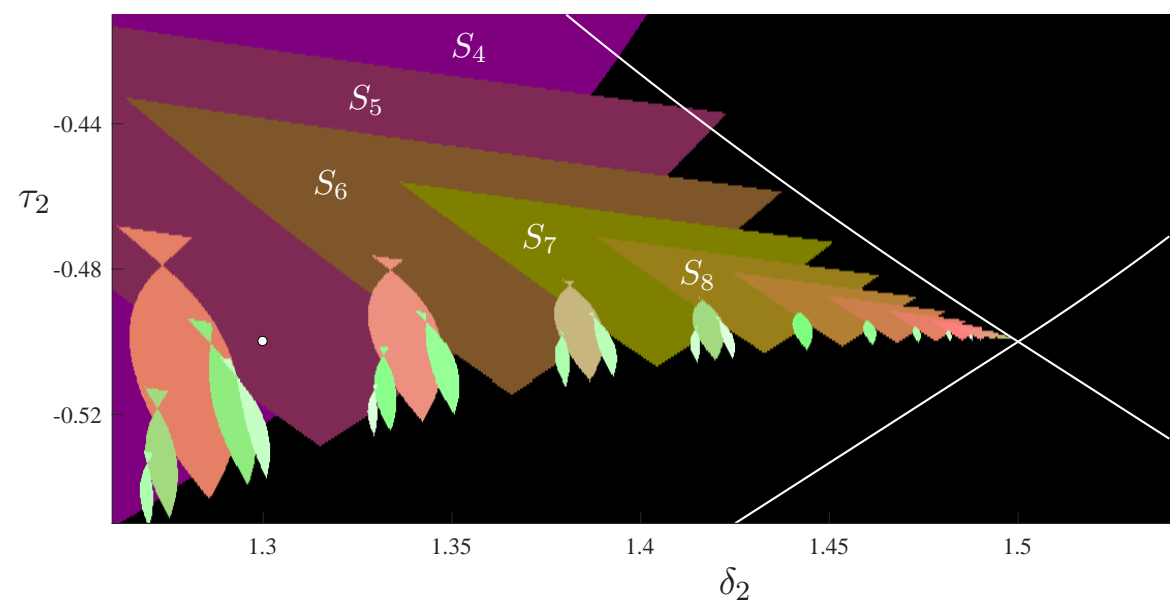

Figure 10: A two-parameter bifurcation diagram of (5.4) with (5.6) using the same conventions as Fig. 8. The white circle indicates the parameter values of Fig. 11,

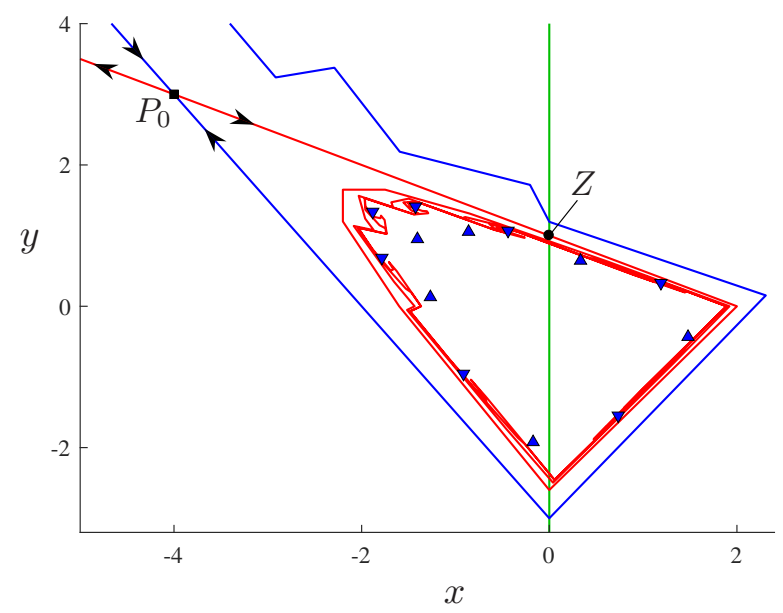

Figure 11: A phase portrait of (5.4) with (5.6) and $\left(\delta_{2}, \tau_{2}\right)=(1.3,-0.5)$ using the same conventions as Fig. 9. The stable periodic solutions (shown with triangles of different orientations) are $1^{4} 2^{2}$ and $1^{5} 2^{2}$-cycles.

Four curves of homoclinic corners intersect at $\left(\tau_{1}, \delta_{1}\right)=\left(-\frac{23}{33}, \frac{13}{66}\right)$. At this point (15.4) has an $\mathcal{X}$-cycle, where $\mathcal{X}=212$, with eigenvalues $\lambda=\frac{4}{11}$ and $\sigma=\frac{13}{6}$ and a subsumed homoclinic connection. Fig. 13 shows a phase portrait at nearby parameter values. Here the conditions of Theorem 3.2 are satisfied using $\mathcal{Y}=12$ (the cyclic permutation of $\mathcal{X}$ has been chosen so that $\left.\mathcal{X}_{0}=\mathcal{Y}_{0}\right)$. Again the regions $S_{k}$ are in accordance with Theorem 3.2. at least for sufficiently large values of $k$ (the region $S_{4}$ has an extra component corresponding to stable $(212)^{4} 22$-cycles). 


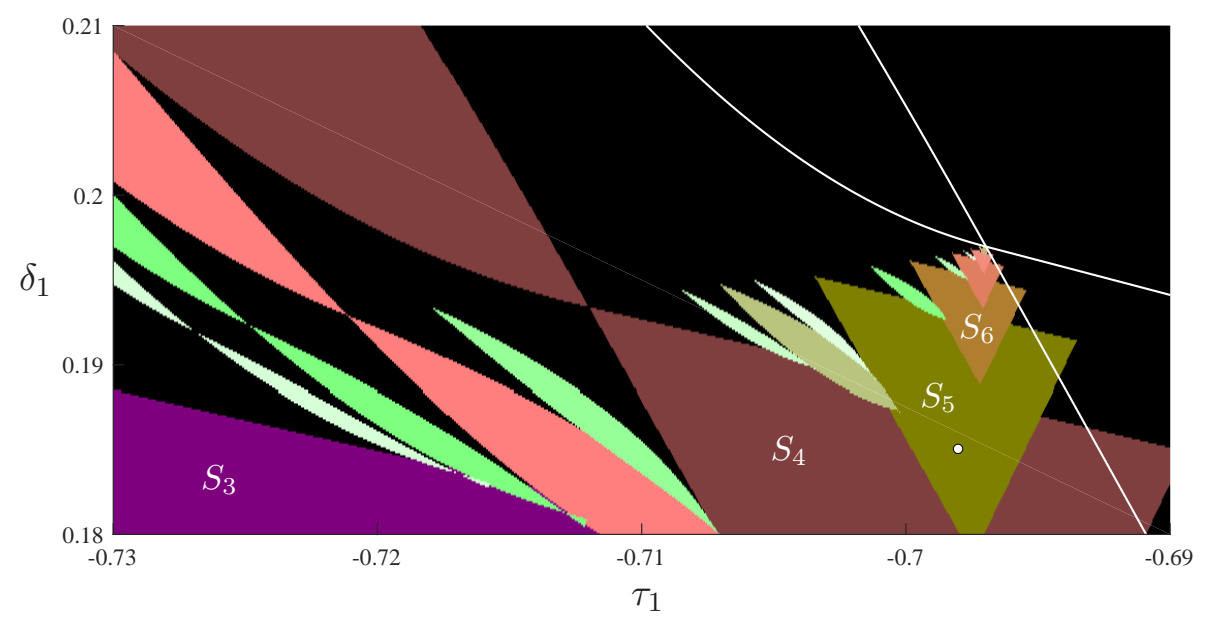

Figure 12: A two-parameter bifurcation diagram of (5.4) with (5.7) using the same conventions as Fig. 8, except periodic solutions have been computed up to period 50. The white circle indicates the parameter values of Fig. 13.

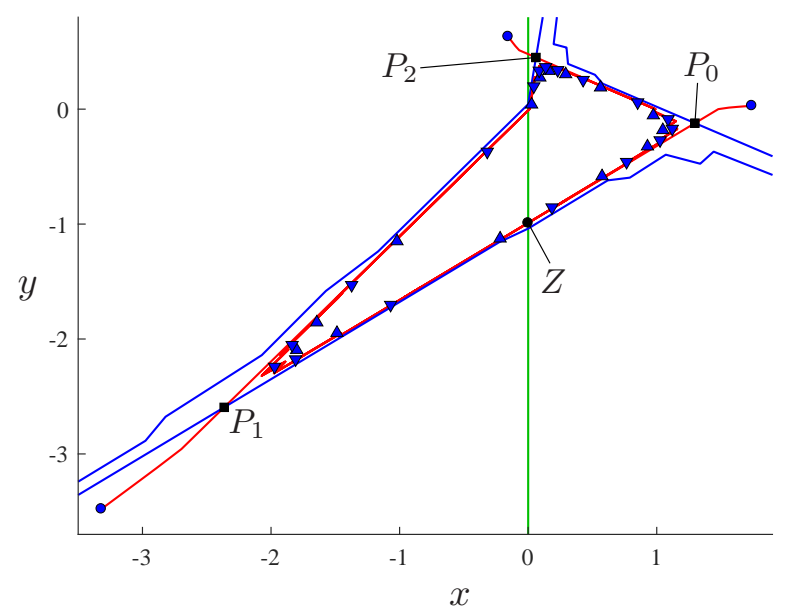

Figure 13: A phase portrait of (5.4) with (5.7) and $\left(\tau_{1}, \delta_{1}\right)=(-0.698,0.185)$ using the same conventions as Fig. 9. Stable $\mathcal{X}^{4} \mathcal{Y}$ and $\mathcal{X}^{5} \mathcal{Y}$-cycles are shown with triangles of different orientations. There also exists a stable 211-cycle shown with circles.

\section{Discussion}

Stable $\mathcal{X}^{k} \mathcal{Y}$-cycles do not exist near codimension-one homoclinic corners [16]. This paper shows that near certain codimension-two homoclinic connections, stable $\mathcal{X}^{k} \mathcal{Y}$-cycles do exist in nearby regions $S_{k}$, Theorem 3.2. To leading order $S_{k}$ is the set $\psi^{-1}\left(\Delta_{k, 0}^{+}\right)$where $\Delta_{k, 0}^{+}$is triangular. This explains, quantitatively, the bulk of the two-parameter bifurcation diagrams shown in Figs. 8, 10, and 12 for three different examples. Each $S_{k}$ overlaps only $S_{k-1}$ and $S_{k+1}$ for large $k$, Proposition 3.3. In such overlaps there exist two stable periodic solutions, as shown in Figs. 9, 11, and 13. 
Near the codimension-three scenario that we also have $\lambda \sigma=1$, for any $N \geq 1$ there exist open regions of parameter space in which $N$ different regions $S_{k}$ overlap [2, 13, 14]. For smooth area-preserving maps the analogous scenario is only codimension-two because the condition $\lambda \sigma=1$ is satisfied automatically [5, 7].

It remains to generalise Theorem 3.2 to higher dimensions, following [19]. It also remains to characterise multi-round periodic solutions. Certainly from the above examples regions where multi-round periodic solutions are admissible and stable appear to exhibit a consistent structure nestled between the $S_{k}$.

\section{Acknowledgements}

The author gratefully acknowledges the assistance of Edward (Zhiyang) Chen regarding calculations of the boundaries of the $S_{k}$.

\section{References}

[1] G.J. Davis. Infinitely many coexisting sinks from degenerate homoclinic tangencies. Trans. Am. Math. Soc., 323(2):727-748, 1991.

[2] Y. Do and Y.-C. Lai. Multistability and arithmetically period-adding bifurcations in piecewise smooth dynamical systems. Chaos, 18:043107, 2008.

[3] S.N. Elaydi. Discrete Chaos with Applications in Science and Engineering. Chapman and Hall., Boca Raton, FL, 2008.

[4] N.K. Gavrilov and L.P. Šil'nikov. On three-dimensional dynamical systems close to systems with a structurally unstable homoclinic curve I. Mat. USSR Sb., 17:467-485, 1972.

[5] M.S. Gonchenko and S.V. Gonchenko. On cascades of elliptic periodic points in two-dimensional symplectic maps with homoclinic tangencies. Regul. Chaotic Dyns., 14(1):116-136, 2009.

[6] S. Gonchenko, D. Turaev, and L. Shilnikov. Homoclinic tangencies of arbitrarily high orders in conservative and dissipative two-dimensional maps. Nonlinearity, 20:241-275, 2007.

[7] S.V. Gonchenko and L.P. Shilnikov. On two-dimensional area-preserving maps with homoclinic tangencies that have infinitely many generic elliptic periodic points. J. Math. Sci., 128(2):2767-2773, 2005.

[8] B. Hao and W. Zheng. Applied Symbolic Dynamics and Chaos. World Scientific, Singapore, 1998.

[9] P. Hirschberg and C. Laing. Successive homoclinic tangencies to a limit cycle. Phys. D, 89:1-14, 1995. 
[10] C. Mira. Embedding of a dim1 piecewise continuous and linear Leonov map into a dim2 invertible map. In G.I. Bischi, C. Chiarella, and I. Sushko, editors, Global Analysis of Dynamic Models in Economics and Finance., pages 337-368. Springer, New York, 2013.

[11] H.E. Nusse and J.A. Yorke. Border-collision bifurcations including "period two to period three" for piecewise smooth systems. Phys. D, 57:39-57, 1992.

[12] J. Palis and F. Takens. Hyperbolicity and sensitive chaotic dynamics at homoclinic bifurcations. Cambridge University Press, New York, 1993.

[13] D.J.W. Simpson. Scaling laws for large numbers of coexisting attracting periodic solutions in the border-collision normal form. Int. J. Bifurcation Chaos, 24(9):1450118, 2014.

[14] D.J.W. Simpson. Sequences of periodic solutions and infinitely many coexisting attractors in the border-collision normal form. Int. J. Bifurcation Chaos, 24(6):1430018, 2014.

[15] D.J.W. Simpson. Border-collision bifurcations in $\mathbb{R}^{n}$. SIAM Rev., 58(2):177-226, 2016.

[16] D.J.W. Simpson. Unfolding homoclinic connections formed by corner intersections in piecewise-smooth maps. Chaos, 26:073105, 2016.

[17] D.J.W. Simpson. The structure of mode-locking regions of piecewise-linear continuous maps: I. Nearby mode-locking regions and shrinking points. Nonlinearity, 30(1):382-444, 2017.

[18] D.J.W. Simpson and J.D. Meiss. Shrinking point bifurcations of resonance tongues for piecewise-smooth, continuous maps. Nonlinearity, 22(5):1123-1144, 2009.

[19] D.J.W. Simpson and C.P. Tuffley. Subsumed homoclinic connections and infinitely many coexisting attractors in piecewise-linear continuous maps. Int. J. Bifurcation Chaos, $27(2): 1730010,2017$. 